\title{
Mechanism of the Electrical Conductivity in Potassium Croconate Violet
}

\author{
Lawrence M. Doane*,$\dagger$ and Alexander J. Fatiadi* \\ National Bureau of Standards, Washington, DC 20234
}

September 9, 1981

\begin{abstract}
Based on crystallographic analysis and results of the solution electronchemistry, a mechanism for electron conduction is proposed.
\end{abstract}

Key words: conductivity; croconates; crystallographic; electrical; electrochemical; mechanism; $\pi$ acceptors; semiconduction.

\section{Introduction}

The search for non-metallic electrical conductors has brought about the synthesis of organic compounds that ordinarily are not thought of as good electrical conductors. For example, ionic crystals $[1]^{l}$ and polymeric solids [2] have been made to behave as good conductors. Among the best-conducting organic materials synthesized are the charge-transfer salts based on the tetrathiofulvalene (TTF)-tetracyanoquinodimethene (TCNQ) skeleton $[1,3]$. In an effort to make additional complexes of higher electrical conductivity, many new donors and acceptors have been prepared. The psuedo-oxocarbon croconates are examples of new $\pi$-electron acceptors that behave as typical semiconductors and exhibit good conductivity when complexed with TTF [4]. We have reported the solution electrochemistry of several croconate salts in $\mathrm{N}, \mathrm{N}$-dimethylformamide (DMF) [5] and in a preliminary communication [6] we briefly discussed an electron conduction mechanism for crystalline croconate salts. Based on crystallographic analysis [7] and results of the solution electrochemistry [5] a detailed conduction mechanism for potassium croconate violet is proposed in this paper.

\section{Discussion}

A general feature of crystalline non-metallic conductors is the formation of molecular stacks in which interplanar separations are van der Waals distances (ca.

\footnotetext{
*Center for Analytical Chemistry, National Measurement Laboratory.

†Present address: IBM Corporation, Dept. 323, Bldg. 970, Essex Jct, VT 05452.

${ }^{1}$ Figures in brackets indicate literature references at the end of this paper.
}

$3.5 \AA)$ [8]. The assumption made is that within this distance the molecular geometry in the stack favors intermolecular orbital overlap. This overlap forms the bridge which permits electron transfer between the molecules of the stack. Generally, the bridgeheads are redox centers. For our purposes, points of closest approach are intermolecular atomic sites that are potentially electrochemically active and are separated by no more than a van der Waals distance. Points of closest approach are therefore the bridgeheads from which intermolecular electron conduction starts or terminates.

\subsection{Anionic crystal structure}

Potassium croconate violet crystallizes with triclinic symmetry [7]. The cyclopentene rings form stacks parallel to the a-axis with an angle of approximately $32^{\circ}$ formed between the plane defined by the cyclopentene ring (ring plane) and the a-axis. A portion of the stack illustrating the two rings of the unit cell along with the rings directly above and below the unit cell are shown in figure 1. Within the unit cell, the perpendicular separation between ring planes is $3.32 \AA$. However, the perpendicular distance between adjacent ring planes of separate but adjoining unit cells is $3.42 \AA$. In addition, adjacent rings are rotated approximately $180^{\circ}$ to the axis of the stack. This rotation is shown clearly in figure $2 a, b$, in which projections normal to the ring plane are illustrated.

\subsection{Points of closest approach}

By viewing ring projections normal to the ring plane, overlapping atomic sites are selected as possible points of closest approach. The atomic sites considered are those 
that exhibit electrochemical activity. From the solution electrochemistry of potassium croconate violet [5], the dicyanomethylene groups and the alkene bond have been shown to be electrochemically active. It is clear

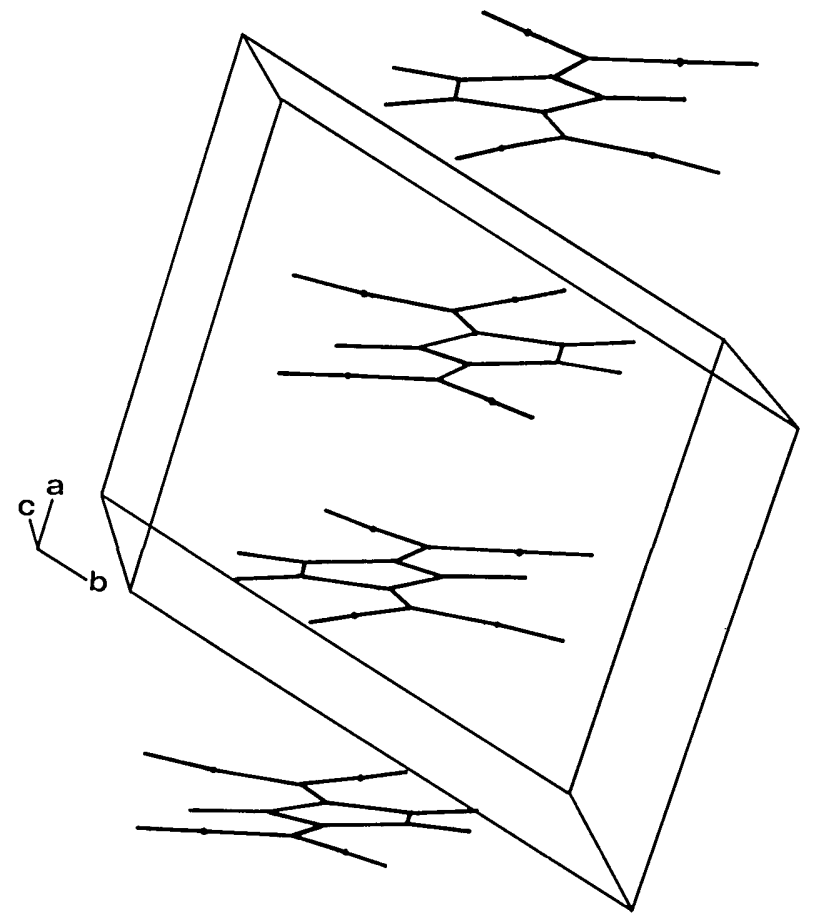

FIGURE 1. View of anionic stack of crystalline potassium croconate violet. from figure $2 b$ that in adjacent rings of separate but adjoining unit cells there is overlap of the dicyanomethylene group of one ring with one of the ring carbon atoms that forms the alkene bond in the other ring (indicated by an asterisk). Similar overlap of adjacent rings within the unit cell is impossible (see fig. 2a). However, overlap of the dicyanomethylene groups of opposing rings is possible. From crystallographic data, atomic distances were determined for the atoms designated by the asterisk in figure $2 \mathrm{a}, \mathrm{b}$. The distances of closest approach, shown in figure 3 , are within the van der Waals distance (3.5 $\AA)$.

\subsection{Proposed electron conduction mechanism}

The solution electrochemistry of croconate violet [5] shows that the only reversible electrochemical reaction occurring in the molecule is the redox process at the 4,5alkene bond. Although in solution electrochemistry the solvation sheath can change significantly after an electron transfer, a general feature of reversible electrochemical processes is the molecule undergoing electron gain or loss changes little structurally. It is apparent then that electron transfer must be initiated and terminated at the alkene bond, since this is the only redox process that leaves the molecule structurally unaltered. In the crystalline croconate salt charge migration may also be cansidered to proceed from one molecule to another by reversible electron transfer at the alkene bond. a)

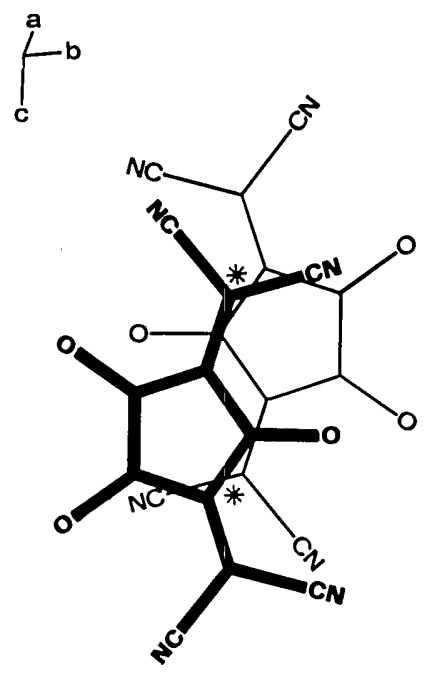

b)

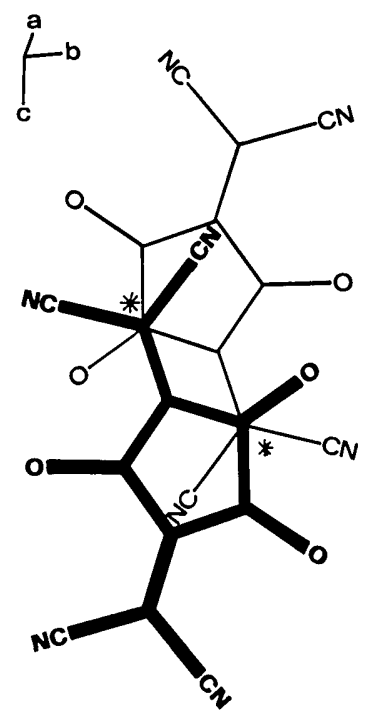

Figure 2. View of projections (normal to the ring plane) of potassium croconate violet: (a) the ring pair within the unit cell and (b) adjacent ring pair of separate but adjoining unit cells. The heavy-lined ring designates the upper of the two croconates. The asterisks designate redox centers of potential overlap. 
Electron migration in crystalline croconate violet is rationalized in the following manner. Consider the stacking arrangement in figure 4 where the cyclopentene rings are parallel and adjacent rings are rotated approximately $180^{\circ}$ to the axis of the stack. The two cyclopentene rings that define the unit cell have their dicyanomethylene groups nearly opposite each other (see fig. 2a). The adjacent ring of the next unit cell is shifted slightly so that one of the dicyanomethylene groups of each ring is opposite the alkene bond of the other ring (see fig. $2 \mathrm{~b}$ ). Electron migration would therefore occur between a di- cyanomethylene group and the alkene bond of adjacent unit cells and between dicyanomethylene groups within the unit cell as illustrated in figure 4 . But, based on the solution electrochemistry of croconate violet the dicyanomethylene group cannot transfer an electron reversibly. However, two rationales can be invoked to explain electron conductivity within the dicyanomethylene group:

(i) Suppose we consider electron movement within the dicyanomethylene group to be a concerted process.

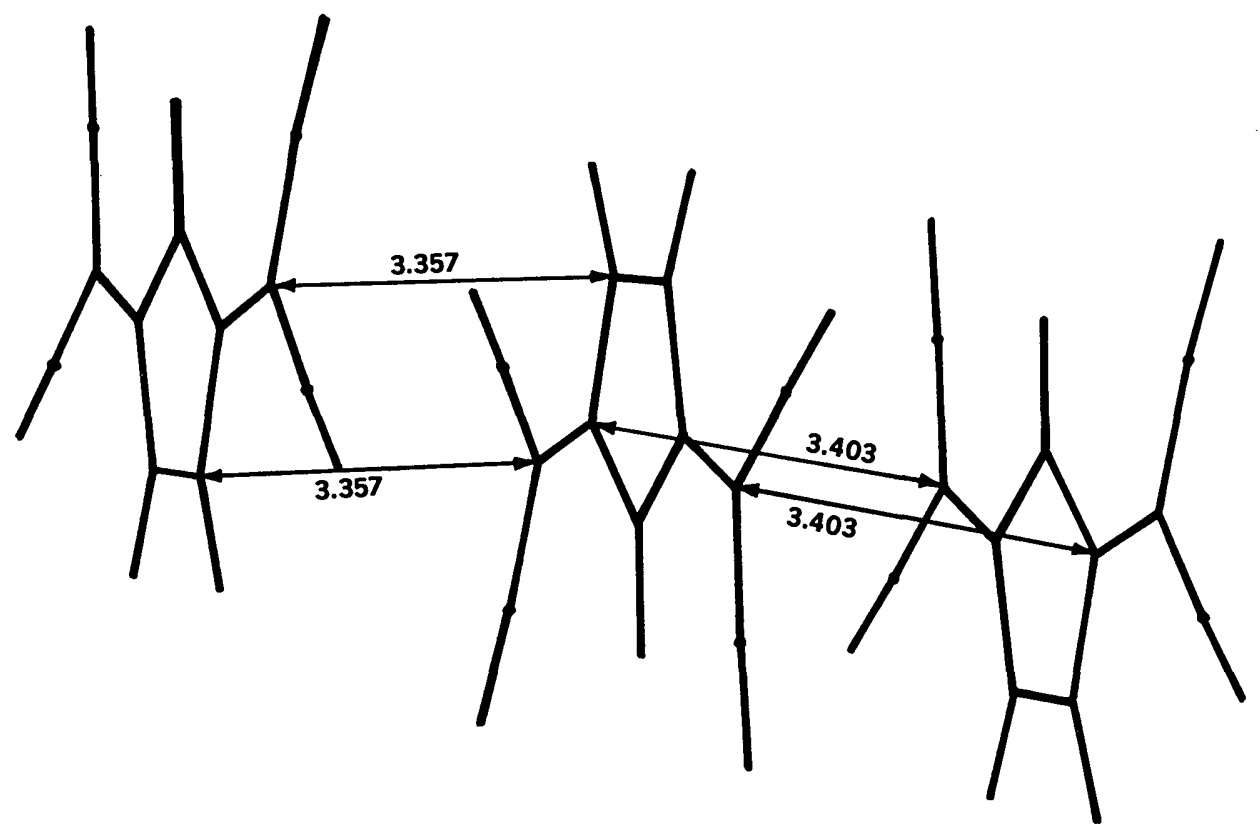

FIGURE 3. View of three stacked croconates indicating atomic distances $(\AA)$ of centers of closest approach.

a)
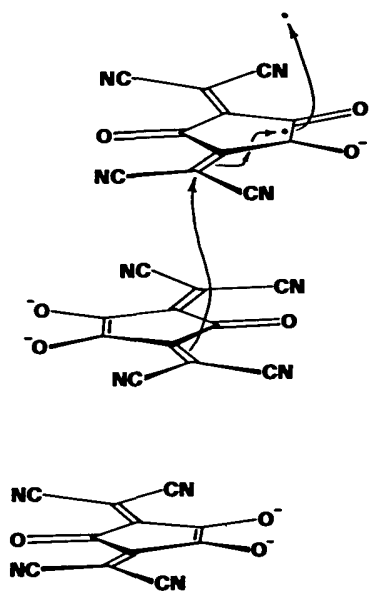

b)
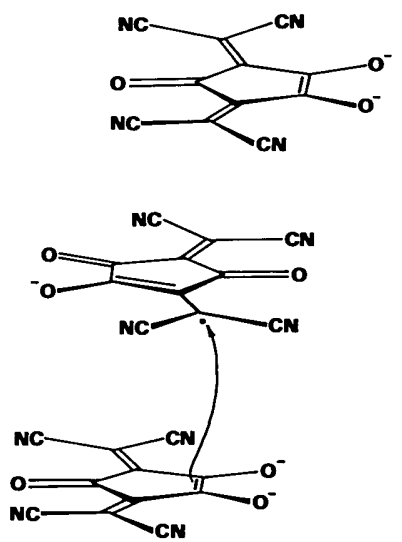

c)
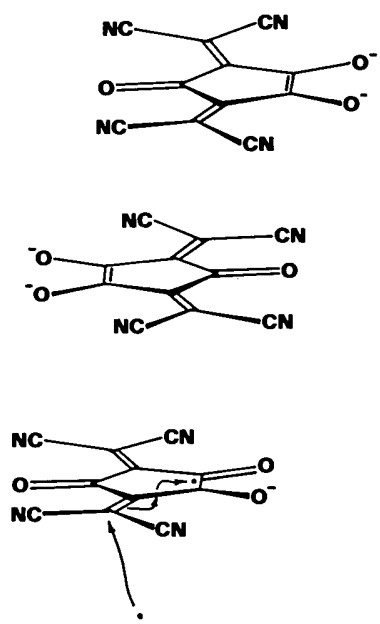

FiGURE 4. Views of three stacked croconates showing proposed: (a) initial electron abstraction and intermolecular electron conduction between dicyanomethylene groups, (b) intermolecular electron conduction between the alkene carbon atom and the dicyanomethylene group (show as a resonance from here), and (c) intramolecular electron conduction between the alkene carbon atom and dicyanomethylene group. 
In this case an electron is injected into one end of the dicyanomethylene group as another electron is simultaneously abstracted from the other end of the dicyanomethylene group. The dicyanomethylene group could then be considered not to have undergone a redox process.

(ii) Resonance structures for the radical anion can be written (Fig. 5) where the dicyanomethylene group participates in delocalizing the unpaired electron. In this case the dicyanomethylene group can participate in intramolecular electron movement in either croconate oxidation state without participating in the actual redox process.

Invoking either explanation for electron conduction within the dicyanomethylene group does not alter the conduction process. The effect is to make the dicyanomethylene group the conduction arm of the molecule while the alkene bond provides the redox mechanism.

One additional comment is in order: since resonance forms can be written for both the dianion and radical anion, there appears to be no migration of resonance in the stack. Mixed valency is therefore not a requisite of electron migration in crystalline croconate violet as has been suggested for other organic conductors. [1].
The authors wish to thank Drs. Alan D. Mighell and William T. Yap for their assistance in obtaining the crystallographic projections and Pam Leech for the artwork.

\section{References}

[1] Perlstein, J. P. Angew. Chem., Int. Ed. Engl., 16 (1977) 519.

[2] Nigrey, P. J.; MacDiarmid, A. G.; Heeger, A. J. J. C. S. Chem. Commun. (1979) 594.

[3] Ferraris, J. P.; Cowan, D. O.; Walatka, V.; Perlstein, J. P. J. J. Am. Chem. Soc., 95 (1973) 948; L. B. Coleman, M. J. Cohen, D. J. Sandman, F. G. Yamagishi, A. F. Garito, and A. J. Heeger, Solid State Commun., 12 (1973) 1125.

[4] Fatiadi, A. J.; in R. West (Ed.), Oxocarbons, Academic Press, New York, 1980, Ch. 4.

[5] Doane, L. M.; Fatiadi, A.J., J. Electroanal. Chem. 135 (1982) 193.

[6] Doane, L. M.; Fatiadi, A. J. Agnew. Chem. (in press).

[7] Hines, V. L.; Mighell, A. D.; Hubbard, C. R.; Fatiadi, A. J. J. Res. Natl. Bur. Stand., 85 (2): 87-97, (1980 March-April).

[8] Soos, Z. G.; Klein, D. L. in R. Foster (Ed.), Molecular Association, Academic Press, New York, 1975, Ch. 1.<smiles>CCC(C#N)(C#N)C1=C([O-])C(=C(C#N)C#N)C(=O)C(=C(C#N)C#N)C1=C(C#N)C#N</smiles>

FIGURE 5. Several possible resonance forms of the croconate violet radical anion. 